DOI: $10.17951 / \mathrm{pjss} / 2016.49 .2 .133$

\title{
KAROLINA HERODOWICZ
}

\section{INFLUENCE OF THE DISTANCE BETWEEN A REFLECTANCE SENSOR AND SOIL SAMPLES WITH DIFFERENT ROUGHNESS ON THEIR SPECTRA}

Abstract. The study assessed the influence of the distance between a reflectance sensor and soil samples with various roughness states (R1- the lowest, R2 - the medium, R3 - the highest roughness state) on their spectra level, under laboratory condition. Studied soil samples were illuminated at three light source zenith angles $\left(\theta_{s}\right.$ equal to $\left.20^{\circ}, 40^{\circ}, 60^{\circ}\right)$ and observed by the sensor to the nadir, from various distances $\left(H_{s}\right)$ from 10 to $54 \mathrm{~cm}$. These dark (the Mollic Gleyic Fluvisol) and light (the Cutanic Stagnic Luvisol) soil materials with their minimum roughness were characterized by diffused reflectance spectra. The relative differences (RD) between the spectra level of soil samples with R1, R2, R3 roughness states and the diffused reflectance level of soil materials were calculated with $1 \mathrm{~nm}$ interval in range of 420-2,300 nm. Higher roughness state and higher $\theta_{s}$, result in higher RD. Thus, for the dark and light soil samples with R3 roughness state and illuminated at $\theta_{s}=60^{\circ}$, the RD are the highest reached 63 and $39 \%\left(H_{s}=54 \mathrm{~cm}\right)$ and reached 77 and $63 \%\left(H_{s}=10 \mathrm{~cm}\right)$, respectively. The spectra level of the soil samples in R1 and R3 roughness states, illuminated at $\theta_{s}=20^{\circ}$ and soil samples with R1 roughness, illuminated at $\theta_{s}=60^{\circ}$, reached a stable level, at a specific $H_{s}$. It means, that a spectra does not significantly change with a further increase $H_{s}$. However, the soil samples in R3 roughness, illuminated at $\theta_{s}=60^{\circ}$ have not reached the stability.

Keywords: spectra level, sensor's distance, soil roughness state, illuminate light source, zenith angle, relative difference

* Adam Mickiewicz University in Poznań, Department of Soils Science and Remote Sensing of Soils, Poznań, Krygowskiego 10 St., 60-101, Poland, e-mail: karolina.herodowicz@amu.edu.pl 


\section{INTRODUCTION}

In recent years remote sensing techniques have become more widespread. These techniques rely on registration of reflected radiation from an analysed surface and they allow to obtain data for: soil monitoring, digital soil mapping, environmental modelling, and precision agriculture (Brown et al. 2006). Also, it allows to measure quantitative information about soil parameters such as soil texture or soil organic matter content (Cécillon et al. 2008). These techniques are used under field or laboratory conditions (Gholizadeh et al. 2013). Visible and near-infrared (VIS-NIR) diffuse reflectance spectroscopy in the wavelength range of 350-2,500 nm has numerous advantages, it is: rapid, non-invasive, reproducible, relatively inexpensive and safe because it replaces the chemical analysis (Reeves 2010). These techniques are divided into: laboratory spectroscopy, portable field spectroscopy, remote spectroscopy i.e. an air- or spaceborne imaging sensor (Stevens et al. 2008).

Nowadays, the proximal soil sensing method (PSS) is used more and more often to get information about soil properties. It is a measuring technique which puts a sensor in contact with a soil surface or in distance less than $2 \mathrm{~m}$ and measures its reflected radiation (Viscarra Rossel et al. 2011; Kuang et al. 2012). Under field conditions, a sensor is installed at a back of the tractors, this method is called on-the-go and tractor-mounted (Adamchuk et al. 2004). However, this measurement is accurate under laboratory conditions due to the possibility of a stable light source and removing the effect of the atmosphere. During field measurements, when a sensor is not in contact with a soil, certain ranges of the spectrum have to be removed due to interference caused by atmosphere (Piekarczyk et al. 2016). Both spectra (from laboratory and field conditions) obtained from the same soil sample, but in different conditions may not be compared directly (Cierniewski, Kuśnierek 2010). Samples of soil under laboratory conditions have to be: airdried, grounded and sieved through a $2 \mathrm{~mm}$ sieve (Soriano-Disla et al. 2014). During the VIS-NIR diffuse reflection spectroscopy measurement, there are certain factors hindering the research i.e. soil moisture, illumination effects and soil roughness (Piekarczyk et al. 2016). Soil moisture greatly affects soil reflectance (Bowers, Hanks 1965; Skidmore et al. 1975). The soil reflectance spectrum rises as the soil becomes drier (Musick, Pelletier 1986).

The reflectance from soil surfaces depends on two directions: the direction from which radiation reaches the surfaces and the direction along which the surfaces are observed by a sensor (Cierniewski et al. 2004). The highest differences in reflected radiation occur along the solar principal plane (SPP). The SPP is a plane where a sensor and sunbeams coming to the surface are located. The highest reflectance from a surface is observed from back scattering directions, at the angle close to the solar zenith angle, while the minimum reflectance is observed from forward scattering directions near the horizon (Cierniewski 1999). 
The soil surface roughness, defined as irregularities occurring on its surface (Thomsen et al. 2015), strongly affects its reflectance. The spectra of soil surface with clods and aggregates are different in comparison with homogenized and smooth soil surface (Cierniewski 1999). Soil aggregates create irregular shapes of soil surface and result in shadowing of a part of the soil surface (Cierniewski 1987). Increase of the soil irregularities result in increase of shaded areas and decrease of the soil reflectance (Matthias et al. 2000). Furthermore, fine-grains have more rounded shapes and greater reflectance spectra, while more cavities and gaps appear in coarse grains, which are traps for incident radiation (Baumgardner et al. 1985; Mikhajlova, Orlov 1986; Cierniewski, Kuśnierek 2010).

Moreover, critical issue of VIS-NIR proximal reflectance spectroscopy measurement is the distance of an instrument from a soil sample. The size of area of the soil sample being measured depends on the angular field of view (FOV). Also, this area depends on the height of a sensor $\left(H_{s}\right)$. Increase of scanned area improves possibilities of achieving representative elementary area (REA), and is done by increasing the distance between sensor and soil surface. This area is defined as the minimum area of the soil surface necessary to conduct reliable measurements. By reaching the REA, a soil parameter becomes independent of the sample size (VandenBygaart, Protz 1998). An analyses of a soil sample in term of spectral reflectance should be large enough, in order to remove deviations from the standard size (Borges et al. 2014).

The aim of this paper is to show the influence of the distance between a reflectance sensor and soil samples with different roughness on their spectra level. The reflectance spectra were obtained by proximal sensing method, under laboratory conditions. The influence was analysed using two different soil materials (dark and light), in four roughness states, illuminated at three light sources zenith angles and observed from a different distance between the sensor and soil samples.

\section{MATERIALS AND METHODS}

Soil materials were collected from two places in Poland, Jeziernik (the dark material) and Złotoryja (the light material) by mixing 10 subsamples taken at the $100 \mathrm{~m}^{2}$ area (Fig. 1). These dark and light materials belong, according to WRB 2007, to the Mollic Gleyic Fluvisol (siltic) and the Cutanic Stagnic Luvisol (siltic), respectively, and contained natural aggregates of different sizes.

Collected soil materials were divided into two parts. The first part consisted of natural aggregates of different sizes, which were used to create soil samples with different roughness states, in the later part of the study. The second part of soil materials was air-dried, and was sieved through a $2 \mathrm{~mm}$ sieve. In this part the soil materials were characterised by their physical and physicochemical properties, such as their texture, $\mathrm{pH}$, the organic carbon and calcium carbonate 


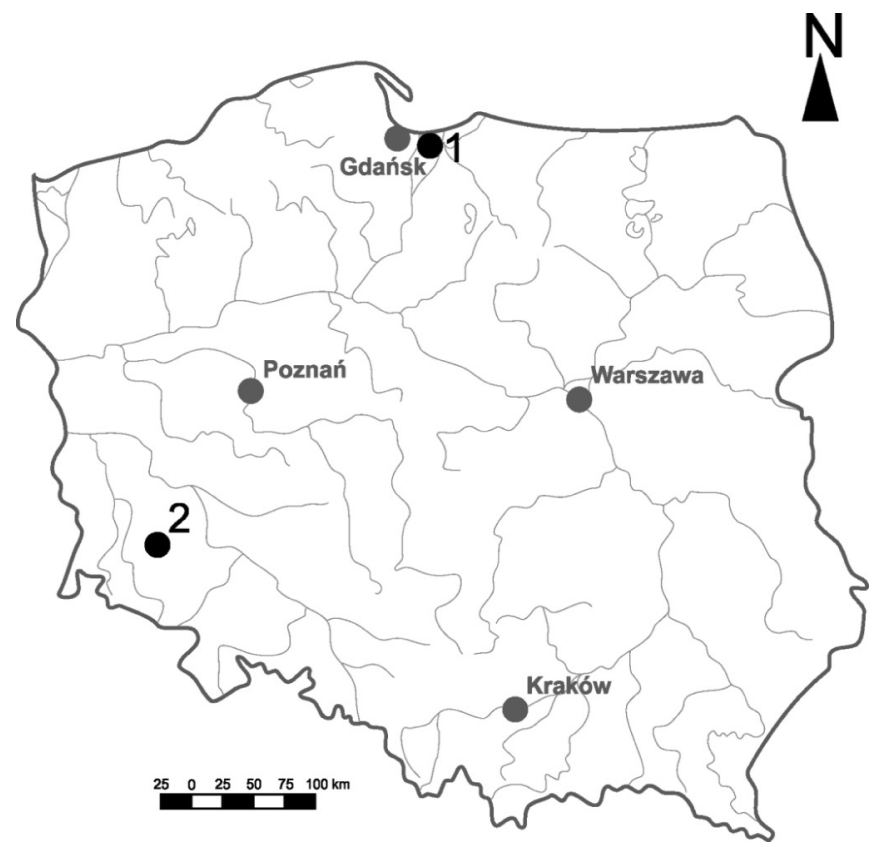

Fig. 1. Location of soil sampling sites, 1 - Jeziernik (the dark material) and 2 - Złotoryja (the light material)

contents and colour according to the Munsell scale. The texture was determined by the sedimentation method according to standard PN 004032. The $\mathrm{pH}$ of the soil was determined in water $(1: 1)$. The organic carbon content was determined using an oxidation titration method on the block mineralization by Nelson and Sommers. The calcium carbonate content was obtained using the volumetric Scheibler method.

Furthermore, these air-dried and sieved soil materials, weighing 10 grams, (labelled as $\mathrm{S}$ - smooth) were used to obtain diffused reflectance spectra. For this purpose, spectroradiometer FieldSpec $₫ 3$ with a Hi-Brite Muglight receptor, produced by the American company ASD Inc. was used. The spectroradiometer is equipped with three detectors, that work in different wavelength ranges: in the visible and near-infrared range (Visible/Near-Infrared - VNIR) from 350 to 1,000 $\mathrm{nm}$, in the range of 1,000-1,830 nm, and 1,830-2,500 nm corresponding to the short-wave part of the infrared radiation (SWIR - Short-Wave Infrared). The instrument recorded of diffused reflectance spectra these soil materials from a distance of a few millimetres and at a constant illumination angle. These measurements were performed twice, for each soil material, after rotating them by $180^{\circ}$. The calibration measurements with Spectralon were performed every 15 minutes.

For each soil material (the dark and the light) soil samples were artificially formed into three roughness states. Parts of the air-dried and sieved soil materials were placed on three circle plastic trays with $28 \mathrm{~cm}$ diameter. The first soil 
sample was created by placing small aggregates, at large distances from each other, on top of these sieved materials, it had the lowest soil roughness (labelled as R1). Next, on the second tray, medium soil aggregates were placed in greater density, on top of sieved materials, creating the medium soil roughness state (R2). On the last tray the largest soil aggregates were placed again on aforementioned sieved materials, creating the highest roughness state (R3).

The shape of surfaces of the artificially prepared soil samples were measured, by placing trays on a table of a laser scanner Konica Minolta VIVID-910 under appropriate lighting conditions of about 500 lx (Vivid 910i Laser Scanner User Manual, 2001-2006). These surfaces were observed by the scanner from 6 directions, by rotating the table by increments of $60^{\circ}$ and from a distance of $1 \mathrm{~m}$ (Fig. 2). Such distance from the laser scanner to soil samples allowed to obtain accuracy of the measurements of $0.1 \mathrm{~mm}$ along axes $\mathrm{X}, \mathrm{Y}$ and $\mathrm{Z}$. These measurements allowed the creation of a digital elevation model (DEM), and calculation of height standard deviation (HSD) parameter of tested surfaces by using TNTMips software. This parameter describes a shape of soil surface used within its delineated basic DEM unit (Marzahn et al. 2012). The calibration procedure was performed, before each measurement, with a special white panel to determine the centre of the layout. The values of HSD were obtained from Świderska's paper (2015).

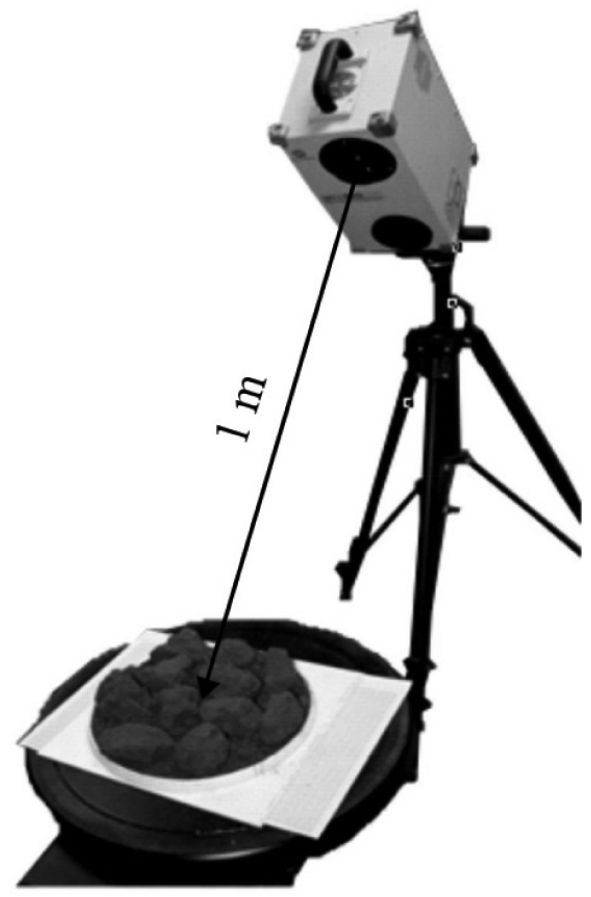

Fig. 2. The soil sample observed from a distance of $1 \mathrm{~m}$ by the laser scanner Konica Minolta 
Each soil sample was measured spectrally using the same spectroradiometer FieldSpec ${ }^{\circledR 3}$. Soil samples were illuminated by light sources, placed at three zenith angles $\left(\theta=20^{\circ}, 40^{\circ}\right.$ and $\left.60^{\circ}\right)$. The sensor was in the zenith position and observed the soil samples with $25^{\circ}$ field of view (FOV), from distances $\left(H_{s}\right): 18,27,36,45$, and $54 \mathrm{~cm}$, at $\theta_{s}=20^{\circ}$, whereas at $\theta_{s}=40^{\circ}$ and $60^{\circ}$ from distances: $10,18,27,36,45$, and $54 \mathrm{~cm}$. The sensor's zenith position represents a position of a sensor during proximal soil sensing method under field conditions. Such position of the sensor mimics the way it is installed vertically at a back of the tractors. The spectroradiometer was calibrated before each measurement, with a white reference spectralon panel (Labsphere, Inc.), keeping the same geometry of light source zenith angle and distance between the sensor and soil samples. The measurements were performed twice for each soil sample after rotating them by $180^{\circ}$.

\section{RESULTS AND DISCUSSION}

The studied soil materials were characterised by selected physical and physicochemical properties. The dark material with the soil colour value of 5 contains 9\% more clay than the light material with the soil colour value of 6 (Table 1). This dark soil material, in comparison with the light one, is characterised by its content of soil organic carbon (SOC) higher by about $1.5 \%$ presence of calcium carbonate $\left(\mathrm{CaCO}_{3}\right)$ and value of $\mathrm{pH}$ lower by about 0.2 and $0.4 \%$, respectively. The light soil material has about 5 and $4 \%$ more sand and silt than the dark soil material, respectively. These properties and the lower SOC content result in the lighter colour of this material.

\section{TABLE 1. SELECTED PHYSICAL AND PHYSICOCHEMICAL PROPERTIES OF THE STUDIED SOIL MATERIALS}

\begin{tabular}{|c|c|c|c|c|c|c|c|}
\hline Soil materials & \multicolumn{3}{|c|}{$\begin{array}{c}\text { Content [\%] } \\
\text { Sand Silt Clay }\end{array}$} & $\mathrm{pH}$ & \multicolumn{2}{c|}{$\begin{array}{c}\text { Content [\%] } \\
\text { SOC CaCO }\end{array}$} & $\begin{array}{c}\text { Colour of dry } \\
\text { soil }\end{array}$ \\
\hline Dark material & 15 & 70 & 15 & 6.44 & 2.84 & 0 & $10 \mathrm{YR} \mathrm{5/3}$ \\
\hline Light material & 20 & 74 & 6 & 6.81 & 1.30 & 0.23 & $10 \mathrm{YR} 6 / 3$ \\
\hline
\end{tabular}

The roughness of the dark and the light soil samples were calculated based on created the surface shape models (Fig. 3a - the dark soil samples and Fig. 3b - the light soil samples) and expressed by HSD parameter (Table 2). 


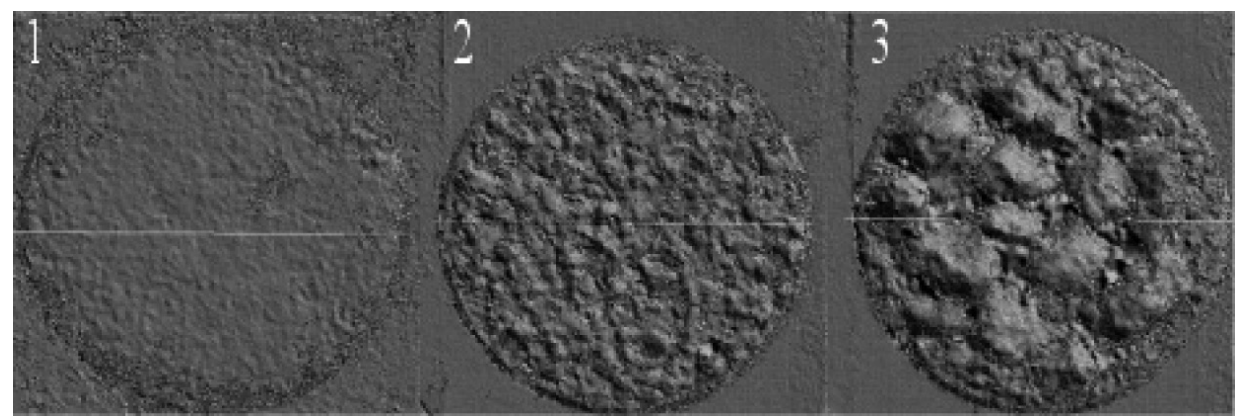

Fig. 3a. Surface shape models of the dark soil samples in three roughness states: 1 - the lowest soil roughness state (R1), 2 - the medium soil roughness state (R2), 3 - the highest roughness state $(\mathrm{R} 3)$

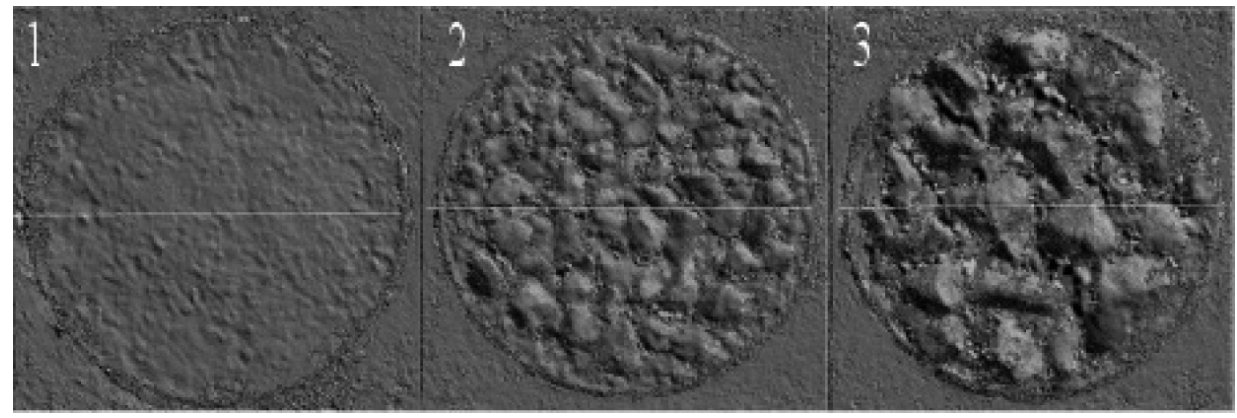

Fig. 3b. Surface shape models of the light soil samples in three roughness states: 1 - the lowest soil roughness state (R1), 2 - the medium soil roughness state (R2), 3 - the highest roughness state (R3)

TABLE 2. VALUES OF HSD PARAMETER OF THE DARK AND LIGHT SOIL SAMPLES IN THREE ROUGHNESS STATES (R1, R2 AND R3)

\begin{tabular}{|c|c|c|c|}
\hline \multirow{2}{*}{ Soil materials } & \multicolumn{3}{|c|}{ HSD [mm] } \\
\cline { 2 - 4 } & R1 & R2 & R3 \\
\hline Dark material & 0.139 & 0.550 & 1.280 \\
\hline Light material & 0.134 & 0.489 & 1.414 \\
\hline
\end{tabular}

The graphs $4 \mathrm{a}$ (the dark soil samples) and $4 \mathrm{~b}$ (the light soil samples) present the reflectance spectrum of sieved materials (S) and spectrum of the each studied soil sample in R1, R2 and R3 roughness states. These soil samples with roughness states were illuminated at three $\theta_{s}$ and were observed at sensor's distances along zenith from various distances.

Wavelength ranges of 350-419 and 2,301-2,500 $\mathrm{nm}$ from all of these spectra were not included in the analysis due to noise. The measurements of the influence of the distance between a reflectance sensor and soil samples with different roughness on their spectra level were presented for just one of the two 

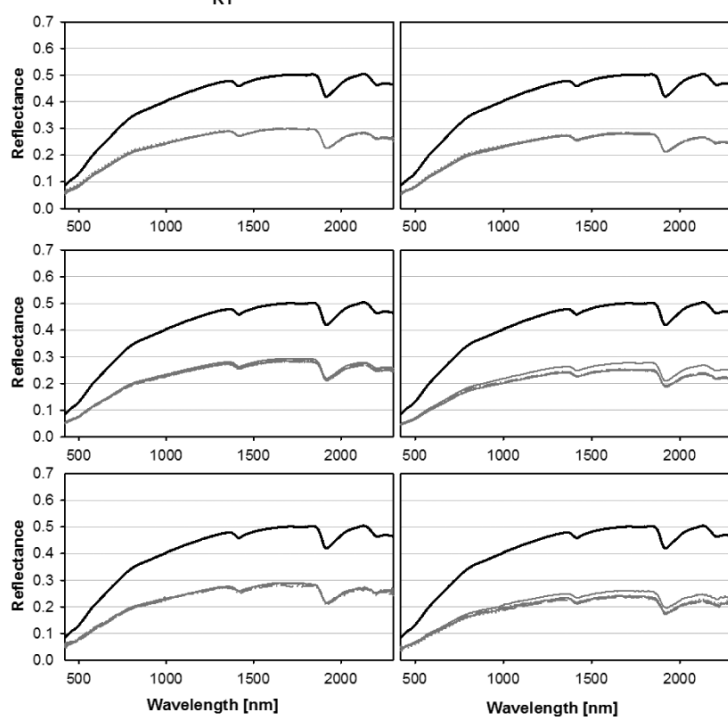

R2

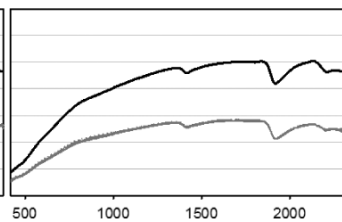

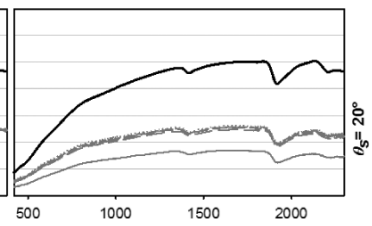

R3

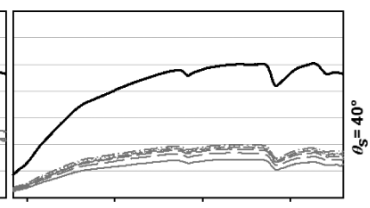

Fig . 4a. The reflectance spectra of the dark soil samples in three roughness states (R1, R2, and R3) and the reflectance spectrum of the sieved

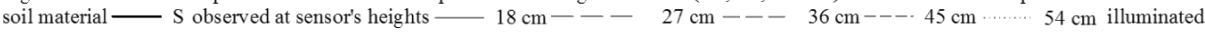

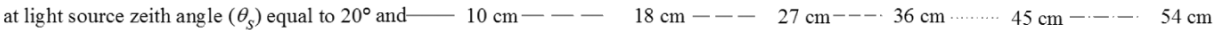
illuminated at $\theta_{s}$ equal to $40^{\circ}$ and $60^{\circ}$
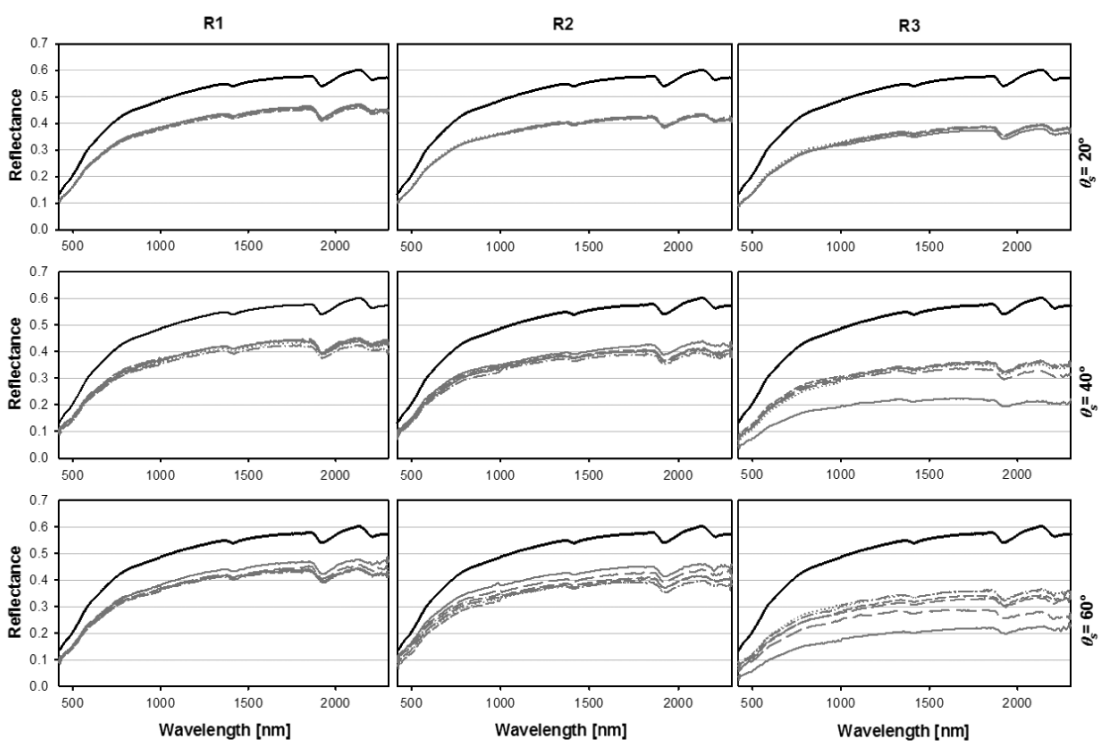

Fig.4b. The reflectance spectra of the light soil samples in three roughness states (R1, R2, and R3) and the reflectance spectrum of the sieved

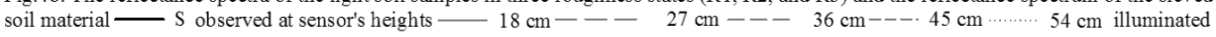

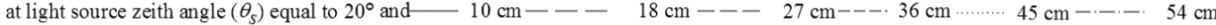
illuminated at $\theta_{S}$ equal to $40^{\circ}$ and $60^{\circ}$ 
measurements due to great similarity of results.

The shape of reflectance spectra of soil samples in R1, R2 and R3 roughness states and spectra $\mathrm{S}$, in both materials (the dark and the light), is similar. The reflectance significantly increases until 1,400 $\mathrm{nm}$ of wavelength, irrespective of roughness states. The dark soil samples with a high content of SOC and clay show a slightly more concave shape in the visible range of wavelengths lower than $700 \mathrm{~nm}$. Whereas the light soil samples with a lower SOC and clay content reveal a convex shape (Courault et al. 1988). Beyond $1400 \mathrm{~nm}$ the increase in the reflectance is lower. There are two minima of the reflectance, located around 1,350 and $1,900 \mathrm{~nm}$, which are an effect of hydroscopic water content, higher for the dark soil samples with a higher clay content.

The greatest differences are in the level of their reflectance spectra between dark and light soil samples in R1, R2 and R3 roughness states and soil materials $(\mathrm{S})$. The level of the reflectance spectrum $\mathrm{S}$, representing a soil material with a minimal roughness, is clearly higher than in the reflectance spectra of soil samples in R1, R2 and R3 roughness states. These differences in the level of reflectance spectra are consistent with earlier results of Cierniewski (1999), showing that an increase in the roughness of soils reduces the level of their spectra. But these differences do not change the shape of their spectra in the wavelength function. The lower level of the spectra of soil samples with higher roughness states is due to shadows created by aggregates and soil particles (Cierniewski 1987). These results obtained in this study confirm earlier reports by Matthias et al. (2000), Cierniewski et al. (2002), Richter et al. (2005), and Wu et al. (2009). In both cases, the soil samples (the dark and the light) show decreasing level of spectral reflectance with increasing roughness and the light source zenith angle.

The spectra level of the soil samples in R1, R2 and R3 roughness states also depends on the $H_{s}$, which has an effect on an observation area. The higher $H_{s}$, the greater the observed area. The higher $H_{s}$ the greater are possibilities of achieving representative elementary area (REA) (VandenBygaart, Protz 1998). The graphs 5a and 5b present the relative differences (RD) between $\mathrm{S}$ spectra level and spectra level of the soil samples in R1, R2 and R3 roughness states in a function of the wavelength with $1 \mathrm{~nm}$ interval in range of 420-2,300 $\mathrm{nm}$. The RD were calculated as:

$$
\operatorname{RD}(\%)=100 \frac{\mathrm{R}_{\mathrm{i}, \mathrm{j}, \mathrm{Hs}}-\mathrm{DR}_{\mathrm{i}}}{\mathrm{DR}_{\mathrm{i}}}
$$

where: $R$ is a reflectance spectrum of the soil sample, $S$ is a reflectance spectrum of the soil material, $i$ is a wavelength, $j$ is a roughness state (R1, R2 and $\mathrm{R} 2), H_{s}$ is sensor's distances.

However, in order to facilitate analysing, the level of the RD of each stud- 

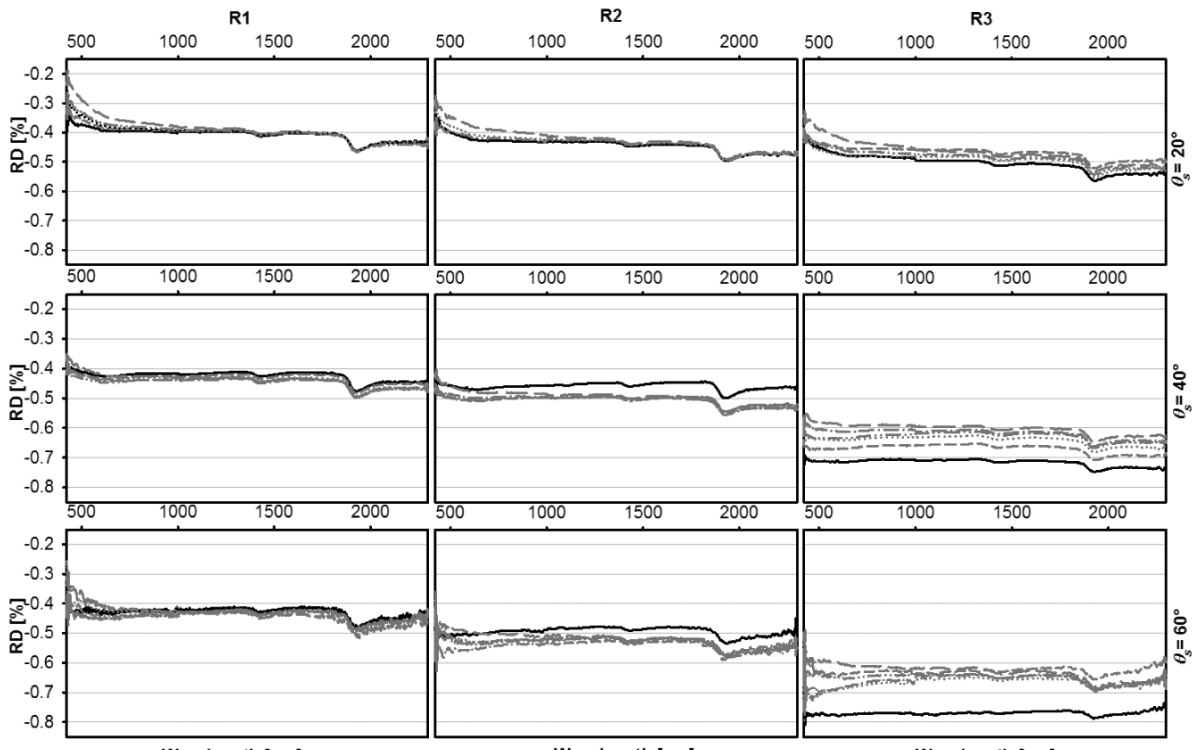

Wavelength [nm]

Wavelength [nm]

Wavelength [nm]

Fig. 5a Relative differences $(\mathrm{RD})$ between the $\mathrm{S}$ reflectance spectrum and reflectance spectra of the dark soil samples in $\mathrm{R} 1, \mathrm{R} 2$, and

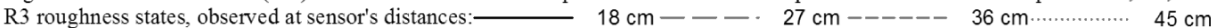
$-\cdot \cdots-\cdot \quad 54 \mathrm{~cm}$ illuminated at light source zenith angle $\left(\theta_{S}\right)$ equal $20^{\circ}$, and $-10 \mathrm{~cm}---18 \mathrm{~cm}$

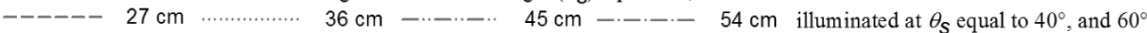
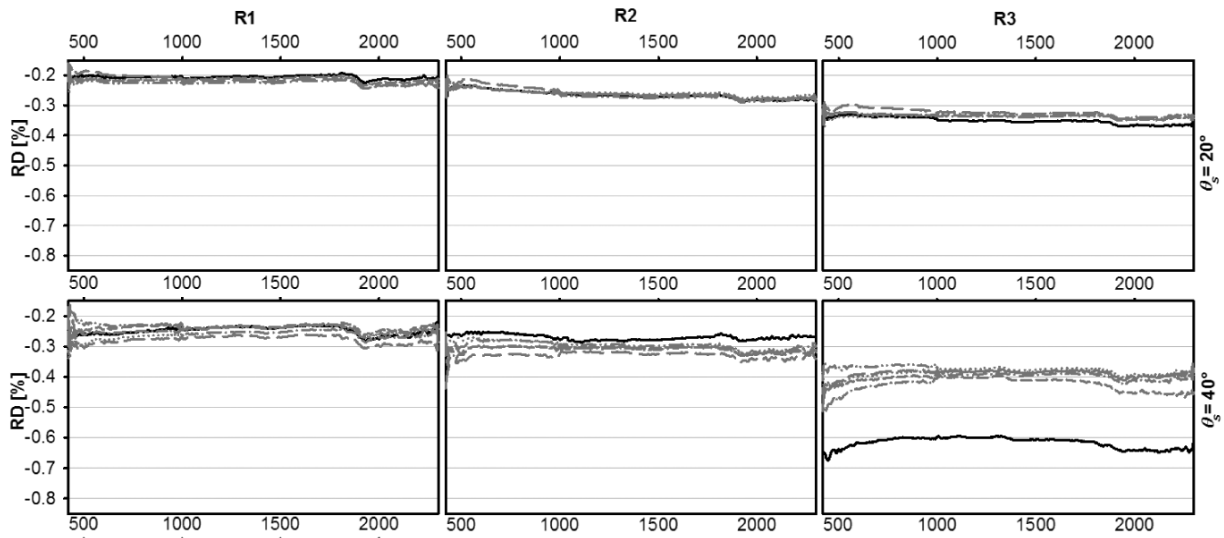

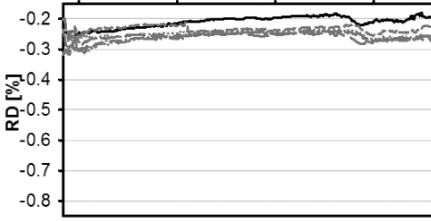

Wavelength $[\mathrm{nm}]$

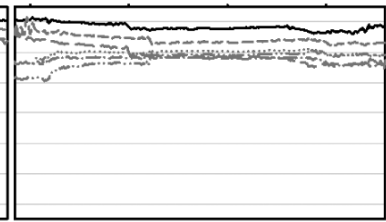

Wavelength [nm]

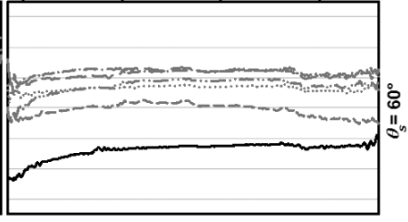

Wavelength $[\mathrm{nm}]$

Fig. 5 b Relative differences $(\mathrm{RD})$ between the $\mathrm{S}$ reflectance spectrum and reflectance spectra of the light soil samples in $\mathrm{R} 1, \mathrm{R} 2$ and $\mathrm{R} 3$ roughness states, observed at sensor's distances: $-18 \mathrm{~cm}-\ldots-27 \mathrm{~cm} \quad--36 \mathrm{~cm} \cdots \cdots \cdots . . .45 \mathrm{~cm}$ $-\cdot-\cdot-\cdot \quad 54 \mathrm{~cm}$ illuminated at light source zenith angle $\left(\theta_{S}\right)$ equal $20^{\circ}$ and $-10 \mathrm{~cm}--18 \mathrm{~cm}---27 \mathrm{~cm}$

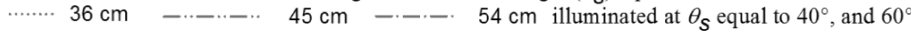


ied soil sample (the dark and the light) was expressed as an average of range $420-2,300 \mathrm{~nm}$.

The RD of the dark soil sample in $\mathrm{R} 1$ roughness state, illuminated at $\theta_{s}=20^{\circ}$, reaching 41 and $40 \%$, at $H_{S}$ equal to 18 and $54 \mathrm{~cm}$, respectively. Meanwhile, the dark soil sample in $\mathrm{R} 3$ roughness state, illuminated at the same $\theta_{s}$, its RD are higher reaching 51 and $48 \%$, for the same $H_{S}$, respectively. The RD are the highest for the soil samples illuminated at $\theta_{s}=60^{\circ}$. Thus, the dark soil sample in R1 roughness states, at this illumination, reaching 44 and $45 \%$, at $H_{S}$ equal to 10 and $54 \mathrm{~cm}$, respectively. The $\mathrm{RD}$ of the dark soil samples in $\mathrm{R} 3$ roughness state, illuminated at the same $\theta_{s}$, reaching in the same order, 77 and $63 \%$, respectively (Table 3 ).

TABLE 3. RD OF THE DARK SOIL SAMPLES IN THREE ROUGHNESS STATES (R1, R2 AND R3), ILLUMINATED AT $\Theta_{S}=20^{\circ}, 40^{\circ}, 60^{\circ}$ AND OBSERVED FROM $H_{S}$ EQUAL TO 18 OR 10 AND 54 CM

\begin{tabular}{cccc}
\hline$\theta_{s} H_{s}$ & $\mathrm{R} 1$ & $\mathrm{R} 2$ & $\mathrm{R} 3$ \\
\hline $20^{\circ} 18 \mathrm{~cm}:$ & $41 \%$ & $45 \%$ & $51 \%$ \\
$54 \mathrm{~cm}:$ & $40 \%$ & $43 \%$ & $48 \%$ \\
\hline $40^{\circ} 10 \mathrm{~cm}:$ & $43 \%$ & $46 \%$ & $72 \%$ \\
$54 \mathrm{~cm}:$ & $44 \%$ & $50 \%$ & $61 \%$ \\
\hline $60^{\circ} 10 \mathrm{~cm}:$ & $44 \%$ & $49 \%$ & $77 \%$ \\
$54 \mathrm{~cm}:$ & $45 \%$ & $53 \%$ & $63 \%$ \\
\hline
\end{tabular}

The RD of the light soil sample in R1 roughness state, illuminated at $\theta_{s}=20^{\circ}$, reaching 21 and $22 \%$, at $H_{S}$ equal to 18 and $54 \mathrm{~cm}$, respectively. The $\mathrm{RD}$ of the light soil sample in $\mathrm{R} 3$ roughness state, illuminated the same $\theta_{s}$, reached in the same order, 35 and $33 \%$, respectively. For the soil sample in R1 roughness state, but illuminated at $\theta_{s}=60^{\circ}$, its RD, reaching 21 and $26 \%$, at $H_{S}$ equal to 10 and 54 $\mathrm{cm}$, respectively. Meanwhile, the RD are the highest for the light soil sample in $\mathrm{R} 3$ roughness, illuminated at the same $\theta_{s}$, reaching in the same order, 63 and $39 \%$, respectively (Table 4 ).

TABLE 4. RD OF THE LIGHT SOIL SAMPLES IN THREE ROUGHNESS STATES (R1, R2 AND R3), ILLUMINATED AT $\Theta_{S}=20^{\circ}, 40^{\circ}, 60^{\circ}$ AND OBSERVED FROM $H_{S}$ EQUAL TO 18 OR 10 AND 54 CM

\begin{tabular}{cccc}
\hline$\theta_{s} \boldsymbol{H}_{s}$ & R1 & $\mathbf{R 2}$ & $\mathbf{R 3}$ \\
\hline $\mathbf{2 0} 18 \mathrm{~cm}:$ & $21 \%$ & $27 \%$ & $35 \%$ \\
$54 \mathrm{~cm}:$ & $22 \%$ & $26 \%$ & $33 \%$ \\
\hline $\mathbf{4 0} 10 \mathrm{~cm}:$ & $25 \%$ & $27 \%$ & $62 \%$ \\
$54 \mathrm{~cm}:$ & $28 \%$ & $33 \%$ & $39 \%$ \\
\hline $\mathbf{6 0}^{\circ} 10 \mathrm{~cm}:$ & $21 \%$ & $22 \%$ & $63 \%$ \\
$54 \mathrm{~cm}:$ & $26 \%$ & $31 \%$ & $39 \%$ \\
\hline
\end{tabular}

Lower $H_{s}$ results in higher $\mathrm{RD}$, especially for soil samples in $\mathrm{R} 3$ roughness 
state. Higher roughness state and higher $\theta_{s}$ also result in higher RD of the studied soil samples. However, a stabilization of the spectra level of the soil samples in $\mathrm{R} 1$ roughness state, illuminated at $\theta_{s}=20^{\circ}$ has been achieved, at sensor's distance equal to $27 \mathrm{~cm}$. The stabilization of spectra level, occurs at a specific $H_{s}$, it means, that a spectra does not significantly change with a further increase of $H_{s}$. The spectra level of the soil samples in R3 roughness, illuminated the same $\theta_{s}$ are characterised by achievement a stable level, at $H_{s}$ equal to $36 \mathrm{~cm}$. Meanwhile, the reflectance spectra level of the soil samples in R1 roughness state, illuminated at $\theta_{s}=60^{\circ}$ have stabilized at $H_{s}$ equal to $45 \mathrm{~cm}$. The spectra reflectance level of the soil samples in R3 roughness state, illuminated the same $\theta_{s}$ have not reached the stability of even the highest $H_{s}$. However, these differences of the spectra level are getting smaller with higher $H_{s}$. Moreover, it has been noted greater RD between successive $H_{s}$ of the light soil samples as compared to the dark soil samples (Wallace 1986).

The spectra reflectance and consequent $\mathrm{RD}$ also depend on the colour value of the soil material. The average level of the dark soil samples, with a higher SOC and clay contents is lower than that of the light soil samples, with lower contents of SOC and clay. The average reflectance of the light material with the roughness $\mathrm{S}$ is higher by $17 \%$ in relation to the dark material. The light soil samples, illuminated at $\theta_{s}=20^{\circ}$ is characterised by a higher spectral reflectance as compared to the dark soil samples, by $36 \%$, in all roughness states, for $H_{s}$ equal $54 \mathrm{~cm}$, respectively. The average reflectance spectra, at $\theta_{s}=40^{\circ}$ of the light soil samples in R1, R2, and R3 are higher, by 36,38 and $47 \%$ as compared to the dark soil samples with similar R states, for the same $H_{s}$, respectively. Whereas at $\theta_{s}=60^{\circ}$ and the same $H_{s}$ these values are the highest, in the same order, by 38 , 43 , and $51 \%$, respectively. According to the previously research conducted by Courault et al. (1988), the colour of soils affects their reflectance spectra. The lighter soil material, the higher their reflectance spectra.

\section{CONCLUSIONS}

1. The level of spectra depends on the colour value of soil materials. Lighter soil material with the colour value of 6 , with a lower SOC by $1.5 \%$ and lower clay contents by $9 \%$, showed a higher reflectance by $17 \%$ in relation to the dark soil material with the colour value of 5 .

2. The spectral reflectance level of the dark soil samples and the light ones, with the smallest roughness $\mathrm{R} 1$ ( $\mathrm{HSD}=0.139$ and $0.134 \mathrm{~mm}$, respectively), the medium roughness 2 ( $\mathrm{HSD}=0.550$ and $0.489 \mathrm{~mm}$, respectively), and the highest roughness $\mathrm{R} 3$ (HSD=1.280 and $1.414 \mathrm{~mm}$, respectively) are clearly lower than their spectra level relation to their soil materials with minimum roughness. Additionally, the illumination light source angle $\left(\theta_{s}\right)$ enhances this effect. High- 
er roughness states and higher $\theta_{s}$ together form more shaded areas and finally the lower spectra level.

3. The distance between a reflectance sensor and the soil samples $\left(H_{s}\right)$ clearly influences the level of their reflectance spectra. The sensor should observe a representative area for studied soil sample. This research shows that higher $\theta_{s}$ and higher roughness of soil samples result in higher relative differences (RD). Therefore, in these cases, higher sensor's height is required. At $H_{s}$ equal to 54 $\mathrm{cm}$, the RD of the dark and the light soil samples in R3 roughness, illuminated $\theta_{s}=60^{\circ}$, reached 63 and $39 \%$, respectively. Whereas at $H_{s}$ equal to $10 \mathrm{~cm}$ the RD of the dark and the light soil samples, in the same conditions, reached 77and $63 \%$, respectively. The spectra level of the soil sample in R1 and R3 roughness states, illuminated at $\theta_{s}=20^{\circ}$, has stabilized at $H_{s}$ equal to 27 and $36 \mathrm{~cm}$, respectively. This stabilization has been also achieved for the soil samples in R1 roughness state, illuminated at $\theta_{s}=60^{\circ}$, at $H_{s}$ equal to $45 \mathrm{~cm}$. However, the reflectance spectra level of the soil samples in R3 roughness, illuminated at the same $\theta_{s}$ have not reached the stability, but smaller differences have been noted at higher $H_{s}$.

4. In the future, studies should be related to identification of the sensor's distance that is necessary to conduct correct research. Thus identification when a rise of a sensor's distance, will not cause changes of the reflectance spectra level.

\section{ACKNOWLEDGEMENTS}

This research was supported by the National Science Centre, Poland, (number of project 2016/21/N/ST10/00308). The author would like to thank Prof. Jerzy Cierniewski and Dr Cezary Kaźmierowski for their valuable comments and suggestions.

\section{REFERENCES}

[1] Adamchuk, V.I., Hummel, J.W., Morgan, M.T., Upadhyaya, S.K., 2004. On-the-go soil sensors for precision agriculture. Computers and Electronics in Agriculture, 44 (1): 71-9.

[2] Baumgardner, M.F, Silva, L.F., Biehl, L.L., Stoner, E.R., 1985. Reflectance properties of soils. Adv. Agron., 38: 1-44.

[3] Borges, J.A.R., Pires, L.F., Costa, J.C., 2014. Representative elementary length to measure soil mass attenuation coefficient. Hindawi Publishing Corporation. The Scientific World Journal, ID 584871.

[4] Bowers, S.A., Hanks, R.J., 1965. Reflection of radiant energy from soils. Soil Sci., 2: 130138.

[5] Brown, D.J., Shepherd, K.D., Walsh, M.G., Mays, M.D., Reinsch, T.G., 2006. Global soil characterization with diffuse reflectance spectroscopy. Geoderma, 32: 273-290.

[6] Cécillon, L., Cassagne, N., Czarnes, S., Gros, R., Brun, J.-J., 2008. Variable selection in near 
infrared spectra for the biological characterization of soil and earthworm casts. Soil Biol. Biochem., 40(7): 1975-1979.

[7] Cierniewski, J., 1987. A model for soil surface roughness influence on the spectral response of bare soils in the visible and near infrared range. Remote Sens. Environ., 23: 97-115.

[8] Cierniewski, J., 1999. Geometrical modeling of soil bidirectional reflectance in the optical domain. Bogucki Scientific Publishers, Poznań.

[9] Cierniewski, J., Verbrugghe, M., Marlewski, A., 2002. Effects of farming works on soil surface bidirectional reflectance measurements and modeling. Int. J. Remote Sens., 23(6): 1075-1094.

[10] Cierniewski, J., Gdala, T., Karnieli, A., 2004. A hemispherical-directional reflectance model as a tool for understanding image distinctions between cultivated and uncultivated bare surfaces. Remote Sens. Environ., 90(4): 505-523.

[11] Cierniewski, J., Kuśnierek, K., 2010. Influence of several soil properties on soil surface reflectance. Quaestiones Geographicae, 29(1): 13-25.

[12] Courault, D., Girard, M.C., 1988. Relationships between the soil composition and the spectral signatures. Proceeding $5^{\text {th }}$ Symposium of the ISSS Working Group on Remote Sensing for Soil Survey, Budapest, Hungary: 178-185.

[13] Gholizadeh, A., Borůvka, L., Saberioon, M., Vasšát, R., 2013. Visible, near-infrared, and mid-infrared spectroscopy applications for soil assessment with emphasis on soil organic matter content and quality: State-of-the-art and key issues. Appl. Spectrosc., 67(12): 13491362.

[14] Kuang, B., Mahmood, H.S., Quraishi, M.Z., Hoogmoed, W.B., Mouazen, A.M., van Henten, E.J., 2012. Sensing soil properties in the laboratory, in situ, and on-line. A review. Adv. Agron., 114: 155-223.

[15] Marzahn, P., Rieke-Zapp, D., Ludwig, R., 2012. Assessment of soil surface roughness statistics for microwave remote sensing applications using a simple photogrammetric acquisition system. ISPRS J. Photogramm., 72: 80-89.

[16] Matthias, A.D., Fimbres, A., Sano, E.E., Post, D.F., Accioly, L., Batchily, A.K., Ferreira, L.G., 2000. Surface roughness effects on soil albedo. Soil Sci. Soc. Am. J., vol. 64(3): 10351041.

[17] Mikhajlova, N.A., Orlov, D.S., 1986. Optical properties of soils and soil components (in Russian). Moskva, Russia, Nauka: 35-38.

[18] Musick, H.B., Pelletier, R.E., 1986. Response of some Thematic Mapper band rations to variation in soil water content. Photogramm. Eng. Remote Sens., 52(10): 1661-1668.

[19] Piekarczyk, J., Kaźmierowski, C., Królewicz, S., Cierniewski, J., 2016. Effects of soil surface roughness on soil reflectance measured in laboratory and outdoor conditions. IEEE Journal of Selected Topics in Applied Earth Observations and Remote Sensing,9(2).

[20] Reeves, III. J.B., 2010. Near-versus mid-infrared diffuse reflectance spectroscopy for soil analysis emphasizing carbon and laboratory versus on-site analysis: Where are we and what needs to be done? Geoderma, 158(1-2): 3-14.

[21] Richter, N., Chabrillat, S., Kaufmann, H., 2005. Preliminary analysis for soil organic carbon determination from spectral reflectance in the frame of the EU project DeSurvey. Pages 96-101 in Proceedings of the $1^{\text {st }}$ International Conference on Remote Sensing and Geoinformation Processing in the Assessment of Land Degradation and Desertification (RGLDD), Trier.

[22] Skidmore, E.L., Dickerson, J.D., Shimmelpfennig, H., 1975. Evaluating surface-soil water content by measuring reflectance. Soil Sci. Soc. Am. Proc. 39(2): 238-242.

[23] Soriano-Disla, J.M., Janik L.J., Viscarra Rossel, R.A., MacDonald, L.M., McLaughlin M.J., 2014. The Performance of Visible, Near-, and Mid-Infrared Reflectance Spectroscopy for Prediction of Soil Physical, Chemical, and Biological Properties. Applied Spectroscopy Reviews, 49(2): 139-186, DOI: 10.1080/05704928.2013.811081

[24] Stevens, A., van Wesemael, B., Bartholomeus, H., Rosillon, D., Tychon, B., Ben-Dor, E., 
2008. Laboratory, field and airborne spectroscopy for monitoring organic carbon content in agricultural soils. Geoderma, 144: 395-404.

[25] Świderska, M., 2015. Soil surface roughness parameters and size of a soil area, in which they are calculated (in Polish), MA thesis, Poznań.

[26] Thomsen, L.M., Baartman, J.E.M., Barneveld, R.J., Starkloff, T., Stolte J., 2015. Soil surface roughness: comparing old and new measuring methods and application in a soil erosion model. Soil Journal, 1: 399-410.

[27] VandenBygaart, A.J., Protz, R., 1998. The representative elementary area (REA) in studies of quantitative soil micromorphology. Geoderma, 89(1999): 333-346.

[28] Viscarra Rossel, R.A., Adamchuk, V.A., Sudduth, K.A., McKenzie, N.J., Lobsey, C., 2011. Proximal soil sensing: A spectrum of opportunities. Adv. Agron., 113: 237-283.

[29] Vivid 910i Laser Scanner User Manual. Japan: Konica Minolta Sensing, 2001-2006.

[30] Wu, C.-Y., Jacobson, A.R., Laba, M., Baveye, P.C., 2009. Accounting for surface roughness effects in the near-infrared reflectance sensing of soils. Geoderma152(1-2): 171-180.

[31] Wallace, K.S., 1986. Surface roughness effects on soil spectral reflectance. University of Arizona, http://hdl.handle.net/10150/275522, p. 144. 